\title{
The Efficacy and Acceptability of Third-Wave Behavioral and Cognitive eHealth Treatments: A Systematic Review and Meta-Analysis of Randomized Controlled Trials
}

\author{
Martin O'Connor \\ Anita Munnelly \\ University College Dublin \\ Robert Whelan \\ Trinity Institute of Neurosciences, Trinity College Dublin, The University of Dublin \\ Louise McHugh \\ University College Dublin
}

\begin{abstract}
eHealth is an innovative method of delivering therapeutic content with the potential to improve access to third-wave behaviural and cognitive therapies. This systematic review and meta-analysis aimed to determine the efficacy and acceptability of third-wave eHealth treatments in improving mental health outcomes. A comprehensive search of electronic bibliographic databases including PubMed, PsycINFO, Web of Science, and CENTRAL was conducted to identify randomized controlled trials of third-wave treatments in which eHealth was the main component. Twenty-one studies were included in the review. Meta-analyses revealed that thirdwave eHealth significantly outperformed inactive control conditions in improving anxiety, depression, and quality-oflife outcomes and active control conditions in alleviating anxiety and depression with small to medium effect sizes. No statistically significant differences were found relative to comparison interventions. Findings from a narrative synthesis of participant evaluation outcomes and meta-analysis of participant attrition rates provided preliminary support for the acceptability of third-wave eHealth.
\end{abstract}

We are thankful to the study authors for providing additional data. This work was supported by the Irish Research Council.

Address correspondence to Martin O'Connor, School of Psychology, Newman Building, University College Dublin, Belfield, Dublin 4; e-mail: martin.o-connor.5@ucdconnect.ie.

0005-7894/@ 2017 Association for Behavioral and Cognitive Therapies. Published by Elsevier Ltd. All rights reserved.
Third-wave eHealth treatments are efficacious in improving mental health outcomes including anxiety, depression, and quality of life, but not more so than comparison interventions. Preliminary evidence from indices of participant evaluation and attrition rates supports the acceptability of these treatments.

Keywords: eHealth; CBT; third wave; systematic review; meta-analysis

THE THIRD GENERATION OR wave of behavioral and cognitive therapies is characterized by a focus on modifying the context and function of private events, rather than modifying their form and frequency (Hayes, Villatte, Levin, \& Hildebrandt, 2011)that is, an emphasis on altering the way in which an individual relates and responds to his or her thoughts, bodily sensations, and emotions, instead of changing the content or intensity of these behaviors. This second-order or contextual change is promoted by targeting processes such as experiential acceptance, defusion, attention to the present moment, and personal values. Moreover, the third wave retains important features of traditional behavioral and cognitive therapies such as functional analysis and skills building (Hayes, Masuda, Bissett, Luoma, \& Guerrero, 2004). Third-wave treatments include acceptance and commitment therapy (ACT; Hayes, 
Strosahl, \& Wilson, 1999), dialectical behavior therapy (DBT; Linehan, 1993), functional analytic psychotherapy (FAP; Kohlenberg \& Tsai, 1991), metacognitive therapy (MCT; Wells, 2009), mindfulness-based cognitive therapy (MBCT; Segal, Williams, \& Teasdale, 2002), schema therapy (Young, 1990), integrative behavioral couple therapy (IBCT; Jacobson \& Christensen, 1996), the cognitivebehavioral analysis system of psychotherapy (CBASP; McCullough, 2000), compassion-focused therapy and compassionate mind training (CFT \& CMT, respectively; Gilbert, 2010).

Systematic reviews and meta-analyses of the evidence for the efficacy of third-wave treatments in improving a range of mental health outcomes have yielded support for ACT (A-Tjak et al., 2015; Bluett, Homan, Morrison, Levin, \& Twohig, 2014; Hacker, Stone, \& MacBeth, 2016; Lee, An, Levin, \& Twohig, 2015), DBT (Kliem, Kröger, \& Kosfelder, 2010; Panos, Jackson, Hasan, \& Panos, 2014), MCT (Normann, van Emmerik, \& Morina, 2014), MBCT (Chiesa \& Serretti, 2011; Piet \& Hougaard, 2011), schema therapy (Jacob \& Arntz, 2013), CBASP (Negt et al., 2016), CFT, and CMT (Leaviss \& Uttley, 2015). These prior reviews examined outcomes including personality disorders, substancerelated disorders, anxiety disorders, and mood disorders, such as major depressive disorder and chronic depression, as well as process measures and satisfaction with life. Cochrane reviews have also found third-wave therapies to be collectively more effective than treatment as usual (Churchill et al., 2013) and equally as effective as other psychological therapies (Hunot et al., 2013) in treating acute depression. However, the quality of the evidence in the aforementioned Cochrane reviews was rated as very low. Furthermore, a meta-analytic review by Öst (2008) synthesized the results of 29 randomized controlled trials of third-wave treatments for a range of problems and found that both ACT and DBT had medium statistically significant effects relative to comparison conditions. Although none of the therapies reviewed by Öst (2008) were judged to fulfill the criteria for empirically supported treatments, a narrative review of subsequent research by Kahl, Winter, and Schweiger (2012) proposed considering all of these treatments, with the exception of CBASP, as empirically supported. In accordance, efforts have been made to improve the dissemination of third-wave treatments.

eHealth provides an innovative method of administering therapeutic content with the potential to increase access to third-wave treatments. eHealth refers to the use of modern information technology and electronic communication resources, including Internet technologies and mobile devices, in the delivery of health care (Oh, Rizo, Enkin, \& Jadad, 2005). Such interventions offer many advantages over traditional methods of delivery. They can reach individuals who are restricted by geographical distance, physical disability, transportation, or financial barriers and provide flexibility of use by allowing clients to access therapeutic content at any time (Proudfoot et al., 2011). Furthermore, eHealth interventions provide high treatment fidelity and consistency of care, while reducing therapist time and waiting lists (Andersson \& Titov, 2014; Cuijpers, van Straten, \& Andersson, 2008). Consequently, third-wave eHealth treatments have been developed and evaluated for a range of problems. Although the reviews cited above support the efficacy of thirdwave treatments delivered in their traditional format, the efficacy of treatments in one format does not necessarily generalize to other modes of intervention delivery (Rosen, Glasgow, Moore, \& Barrera, 2015).

A systematic review by Cavanagh, Strauss, Forder, and Jones (2014) meta-analytically synthesized the results of 15 randomized controlled trials of mindfulness and acceptance-based self-help interventions and found small statistically significant benefits on anxiety $(g=0.34)$ and depression $(g=0.37)$ outcomes relative to control conditions. Similarly, a recent meta-analytic review of 15 randomized controlled trials by Spijkerman, Pots, and Bohlmeijer (2016) also found that online mindfulness-based interventions (MBIs) were superior to control conditions in improving mental health outcomes, with small to medium statistically significant benefits on anxiety $(g=0.22)$, depression $(g=0.29)$, and well-being $(g=$ 0.23 ). Although previous systematic reviews and meta-analyses have investigated the efficacy of thirdwave treatments delivered in their traditional format (Churchill et al., 2013; Hunot et al., 2013; Öst, 2008) and examined the effectiveness of mindfulness and acceptance-based self-help interventions (Cavanagh et al., 2014), as well as online MBIs in improving mental health (Spijkerman et al., 2016), to date there has not been a review of the evidence base for thirdwave behavioral and cognitive eHealth therapies. The steady increase in citations of "third wave" in the scientific literature (Dimidjian et al., 2016) and proliferation of eHealth interventions delivering this therapeutic content (Pierce, Twohig, \& Levin, 2016) underscores the importance of systematically reviewing the evidence for the efficacy and acceptability of third-wave eHealth treatments.

The primary objective of this systematic review and meta-analysis of randomized controlled trials was to determine the efficacy of third-wave eHealth treatments in improving mental health outcomes including anxiety, depression, and quality of life. Moreover, given that a study's type of comparison 
condition has been identified as a moderating factor in meta-analyses (Grist \& Cavanagh, 2013), the effects of the treatments were evaluated relative to inactive, active control conditions and comparison interventions. In addition, potential moderators of the effects of third-wave eHealth-including type of intervention, study population, and risk of biaswere also investigated. The secondary objective was to elucidate the acceptability of third-wave eHealth treatments as measured by indices of participant evaluation, including treatment satisfaction and credibility, as well as participant attrition rates.

\section{Method}

This systematic review and meta-analysis was conducted in accordance with a review protocol and adhered to the Preferred Reporting Items for Systematic Reviews and Meta-Analyses (PRISMA; Moher, Liberati, Tetzlaff, Altman, \& PRISMA Group, 2009).

\section{ELIGIBILITY CRITERIA}

\section{Types of Studies}

Randomized controlled trials were published in English-language peer-reviewed journals reporting on the efficacy of third-wave eHealth treatments in improving anxiety, depression, or quality-of-life outcomes relative to inactive (i.e., no treatment or wait-list), active control conditions and/or comparison interventions. Active control conditions were designed to control for the nonspecific effects of attention and activity. Studies with a dismantling or additive design, comparing the efficacy of components of a third-wave treatment, were excluded.

\section{Types of Participants}

Individuals participated in trials investigating the efficacy of third-wave eHealth treatments, with no exclusions on the basis of age, sex/gender, ethnicity, or morbidity.

\section{Types of Intervention}

Third-wave treatments, including ACT, DBT, FAP, MBCT, MCT, schema therapy, CFT, CBASP, CMT, or IBCT, where the main component of the intervention was delivered via modern information technology and electronic communication resources, including computer, Internet, mobile phone, personal digital assistant, smartphone, or tablet application were eligible for inclusion in the review. In accordance with the previous systematic reviews and metaanalyses of third-wave treatments (Churchill et al., 2013; Hunot et al., 2013; Öst, 2008), mindfulnessbased stress reduction (MBSR; Kabat-Zinn, 1990) was not included in the current review, nor were MBIs that did not explicitly describe their therapeutic approach as one of the aforementioned treatments.

\section{Types of Outcome Measures}

Valid and reliable measures of anxiety, depression, and quality-of-life outcomes were reported at posttreatment. Where two or more measures of the same construct were administered in a single study, the primary outcome measure was selected. Secondary outcomes of interest were indices of the acceptability of third-wave eHealth treatments, including selfreport measures of treatment satisfaction and credibility, as well as participant attrition rates.

\section{INFORMATION SOURCES}

Studies were identified by searching electronic bibliographic databases including PubMed, PsycINFO, Web of Science, and the Cochrane Central Register of Controlled Trials (CENTRAL) for articles published before October 23, 2015. The reference lists of all selected articles were screened for eligible studies as were relevant review articles identified in the searches. In addition, an expert in the topic area was contacted to identify eligible studies. Trial registries including ClinicalTrials.gov and Nederlands Trial Register were searched for study protocols to assess reporting bias in included studies. Seven study authors were contacted for further information and clarification where insufficient data were reported, six of which provided the requested data.

\section{SEARCH STRATEGY}

Key search terms relating to third-wave behavioral and cognitive eHealth treatments were combined with Boolean operators and Medical Subject Headings $(\mathrm{MeSH})$, where appropriate. The full electronic search strategies used for PubMed, PsycINFO, Web of Science, and CENTRAL are reported in a supplementary file. Database-specific filters for "English language" and "humans" were used to refine the scope of the search results.

\section{STUDY SELECTION}

Two reviewers (MOC and AM) independently screened the titles and abstracts of articles identified in the searches. Full-text papers of all potentially eligible studies were retrieved and the inclusion criteria were applied to each study independently by the two reviewers using standardized forms. Discrepancies between the reviewers were resolved through consensus.

\section{DATA COLLECTION PROCESS}

Data were extracted from each included study by one reviewer (MOC) using a prepiloted data extraction form and checked for accuracy by a second reviewer (AM). Where a single study was published in multiple reports, information from all of the publications was synthesized and considered as one source. 


\section{DATA ITEMS}

Information was extracted from each included study on author, publication year, study population, sample size, percentage of females, mean age, outcome measure(s), intervention, comparator, measure(s) of acceptability, attrition rate in each trial arm, follow-up, and method of statistical analysis.

\section{RISK OF BIAS IN INDIVIDUAL STUDIES}

The Cochrane Collaboration's tool for assessing risk of bias in randomized trials (Higgins, Altman, \& Sterne, 2011) was used to ascertain the validity of the included studies. The tool covers seven evidencebased domains of bias: random sequence generation, allocation concealment, blinding of participants and personnel, blinding of outcome assessment, incomplete outcome data, selective outcome reporting, and other bias. Assessments for each domain comprise a judgment of risk of bias and the support for that judgment. Judgments are expressed as high risk, low risk, or unclear risk of bias. Risk of bias assessments were conducted by two reviewers (MOC and LM) independently. Discrepancies between the reviewers were resolved through consensus.

\section{SUMMARY MEASURES}

Hedges's $g$ effect size (ES) was computed using Comprehensive Meta-Analysis software Version 3 to estimate the effects of third-wave eHealth treatments relative to comparators on anxiety, depression, and quality-of-life outcomes at posttreatment. Hedges's $g$ provides an unbiased estimate by correcting for the tendency of Cohen's $d$ to overestimate ES in small samples (Borenstein, Hedges, Higgins, \& Rothstein, 2009). ES estimates were interpreted in accordance with Cohen (1988): 0.2 represents a small effect, 0.5 a medium effect, and 0.8 a large effect. In addition, odds ratio was computed to investigate the ratio of the odds of participant attrition in third-wave eHealth groups relative to comparator conditions. A power analysis revealed that a sample size of 146 participants is required to detect the difference in attrition rates suggested by the most relevant metaanalytic review that investigated participant dropout $(16 \%$ vs. $37 \%$; OR $=3.08$; Spijkerman et al., 2016). In accordance, the current meta-analyses all had $100 \%$ power to detect this estimated ES at $\alpha=.05$.

Interrater agreement was defined as both independent reviewers reporting an identical decision. The percentage of interrater agreement for study selection, data extraction, and risk of bias assessments was calculated by dividing the number of agreements by the sum of agreements and disagreements, and multiplying the product by 100 . The percentage of interrater agreement for study selection, data extrac- tion, and risk of bias assessments was $89.2 \%, 100 \%$, and $99.3 \%$, respectively.

\section{PLANNED METHODS OF ANALYSIS}

Meta-analyses were performed to statistically synthesize the results of the included studies. Estimates of the effects of third-wave eHealth treatments on anxiety, depression, and quality-of-life outcomes were calculated relative to inactive, active control conditions, and comparison interventions. Given the variability in populations and interventions among included studies, these summary effect measures and their $95 \%$ confidence intervals were calculated using random effects models. The random effects model assumes that the studies included in a meta-analysis come from different populations with different average treatment effects (Field \& Gillett, 2010). Secondary analyses were carried out to investigate potential differences in participant attrition between third-wave eHealth and inactive, active control and comparison intervention conditions. Mixed-effects subgroup analyses were conducted to investigate differences in overall ES as a function of type of intervention. In line with Davies, Morriss, and Glazebrook (2014), interventions were classified as universal, selectivel indicated, or treatment. Universal interventions did not involve screening for mental health symptomology, and selective or indicated programs targeted individuals with an above-average level of symptomology or risk of developing a disorder, while treatment interventions targeted individuals with a diagnosed mental disorder (Graber \& Sontag, 2009).

The heterogeneity between studies in each metaanalysis was assessed by visually inspecting forest plots and conducting $Q$ tests for heterogeneity. The degree of heterogeneity was measured using the $I^{2}$ statistic and interpreted in accordance with Blundell (2014): 25\% represents low heterogeneity, 50\% moderate heterogeneity, and $75 \%$ high heterogeneity. Heterogeneity was investigated by performing sensitivity analyses, excluding studies with high standardized residuals from the analyses.

\section{RISK OF BIAS ACROSS STUDIES}

Random effects meta-regression analyses were conducted to investigate the moderating effects of the domains of bias on ES. In accordance with Higgins et al. (2011), studies with high or unclear risk of bias were compared with those at low risk of bias. In meta-regression analyses where a study included more than one comparison condition, the most common comparator across studies was chosen (Cavanagh et al., 2014; Spijkerman et al., 2016). In addition, funnel plots were visually inspected and Duval and Tweedie's (2000) trim-and-fill procedure 
was conducted to detect the presence of publication bias across studies and assess its impact on the metaanalyses.

\section{Results}

\section{STUDY SELECTION}

A total of 6,098 citations were identified through searches of electronic bibliographic databases (PubMed, $n=971$; PsycINFO, $n=572$; CENTRAL, $n=204$; and Web of Science, $n=1,311$ ), reference lists of previous reviews $(n=1,595)$, and relevant articles $(n=1,445)$. After detecting duplicates and screening titles and abstracts for relevance, the full texts of 224 potentially eligible articles were obtained for further assessment. The inclusion criteria were applied to all full-text papers and 18 articles were selected for inclusion. A further 3 articles that met the inclusion criteria were identified through contacting an expert in the topic area. Consequently, 21 studies were included in the systematic review (see Figure 1 for diagram of study selection). The characteristics of the 21 included studies are summarized in Table 1.

\section{STUDY CHARACTERISTICS}

\section{Participants}

Studies included a total of 3,176 participants. Sample sizes ranged from 38 to 1,002 . The mean age of participants in the studies ranged from 18.4 to 52.8 years, and the proportion of females ranged from
43.4 to $100 \%$. Nine studies were recruited from populations with somatic illnesses including chronic pain $(n=4)$, irritable bowel disease $(n=3)$, obesity $(n=1)$, and tinnitus $(n=1)$. The third-wave eHealth treatments evaluated in each of these studies aimed to reduce the disabling influence of the respective somatic illness and improve participants' functioning. Individuals with psychological illnesses were recruited in seven studies: depression $(n=6)$ and binge-eating disorder $(n=1)$. The primary outcome in these studies were assays of the aforementioned psychological illnesses. Nonclinical populations were sampled in the remaining five studies. The interventions administered to these nonclinical populations aimed to decrease anxious and depressive symptomology $(n=3)$ and procrastination $(n=1)$, as well as increase self-compassion $(n=1)$.

\section{Interventions}

Nine studies investigated the efficacy of eHealth interventions based on ACT: eight were Internetdelivered and one was smartphone based. Therapist guidance was provided in eight of these studies (Buhrman et al., 2013; Hesser et al., 2012; Kristjánsdóttir et al., 2013; Lappalainen et al., 2014; Lappalainen, Langrial, Oinas-Kukkonen, Tolvanen, \& Lappalainen, 2015; Pots et al., 2015; Trompetter, Bohlmeijer, Veehof, \& Schreurs, 2015; Weineland, Arvidsson, Kakoulidis, \& Dahl, 2012), the duration

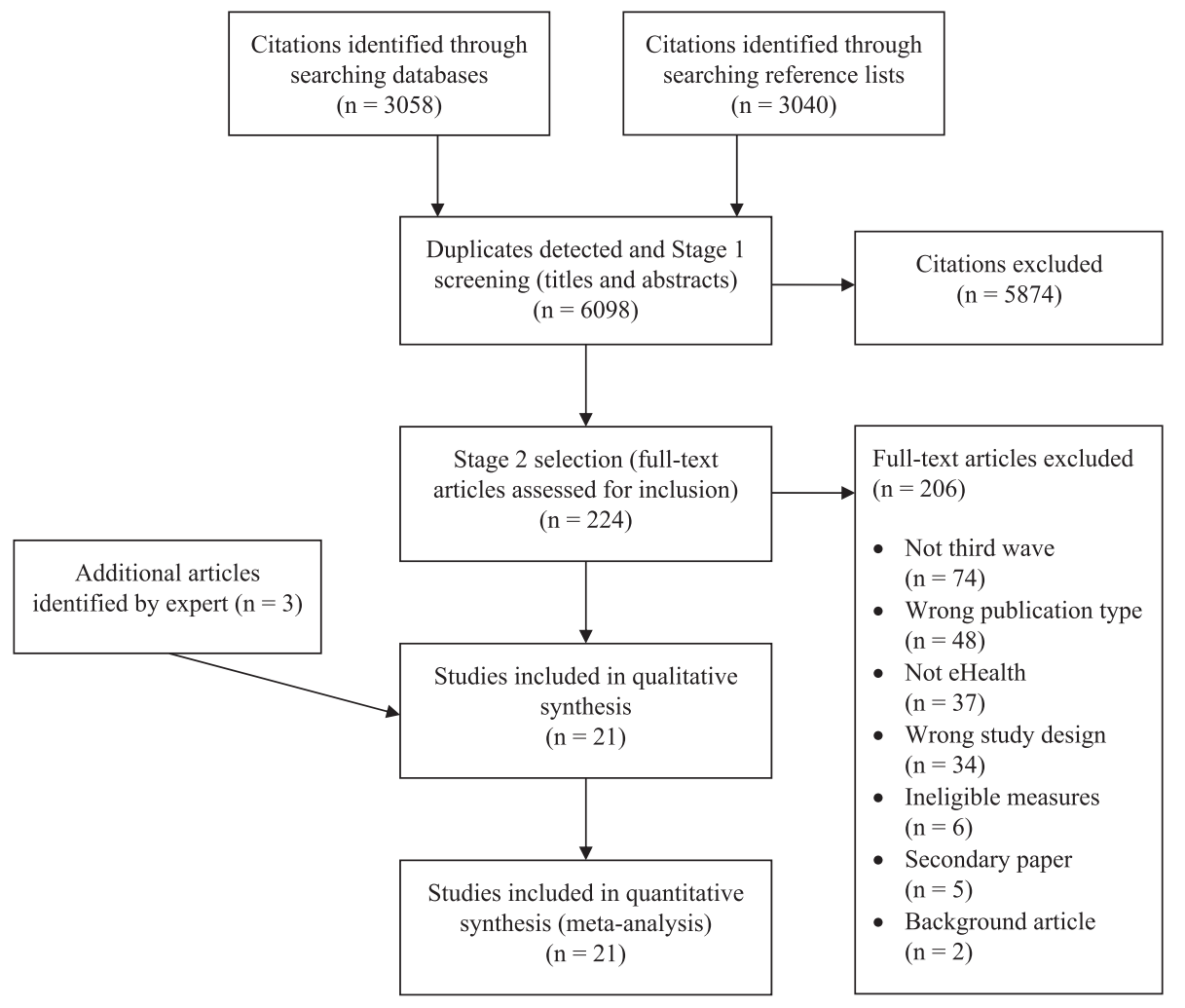

FIGURE I Flow diagram of study selection. 
Table 1

Characteristics of Included Studies

\begin{tabular}{|c|c|c|c|c|c|c|c|c|}
\hline Study & Study population & $\begin{array}{l}\text { Sample } \\
\text { size }\end{array}$ & $\begin{array}{l}M \\
\text { age }\end{array}$ & $\%$ female & Intervention & Type & Comparator & Follow-up \\
\hline Braithwaite \& Fincham, 2007 & Young adults in romantic relationships & 91 & NS & 59 & C-CBASP & $U$ & Active; intervention & None \\
\hline Buhrman et al., 2013 & Chronic pain patients & 76 & 49.1 & 59 & $\mathrm{I}-\mathrm{ACT}$ & $\mathrm{S} / \mathrm{l}$ & Active & 6 months \\
\hline Carlbring et al., 2013 & Adults diagnosed with major depression & 80 & 44.4 & 82.5 & I-BA, I-ACT & $\mathrm{T}$ & Inactive & 3 months \\
\hline Cukrowicz \& Joiner, 2007 & College students & 238 & 19.2 & 71 & C-CBASP & $U$ & Active & None \\
\hline Dowd et al., 2015 & Adults experiencing chronic pain & 124 & 44.5 & 90.3 & I-MBCT & $\mathrm{S} / \mathrm{l}$ & Intervention & 6 months \\
\hline Glick \& Orsillo, 2015 & College students & 118 & 21.1 & 59 & I-ABBT & $U$ & Intervention & None \\
\hline Hesser et al., 2012 & Adults distressed by tinnitus & 99 & 48.5 & 43.4 & $\mathrm{I}-\mathrm{ACT}$ & $S / l$ & Active; intervention & 12 months \\
\hline Kelly \& Carter, 2015 & Adults diagnosed with binge-eating disorder & 41 & 45 & 82.9 & I-CFT & $\mathrm{T}$ & Inactive; intervention & None \\
\hline Kivi et al., 2014 & Adults diagnosed with major depression & 79 & 36.6 & 66 & I-BA, I-ACT & $\mathrm{T}$ & Intervention & None \\
\hline Kristjánsdóttir et al., 2013 & Women experiencing chronic pain & 140 & 44.2 & 100 & S-ACT & $\mathrm{S} / \mathrm{l}$ & Inactive & 5 months \\
\hline Lappalainen et al., 2014 & Adults experiencing depressive symptoms & 38 & 44.6 & 68 & $\mathrm{I}-\mathrm{ACT}$ & $\mathrm{S} / \mathrm{l}$ & Intervention & 6 and 18 months \\
\hline $\begin{array}{l}\text { Lappalainen, Langrial, Oinas-Kukkonen, Tolvanen, } \\
\text { \& Lappalainen, } 2015\end{array}$ & Adults experiencing depressive symptoms & 39 & 51.9 & 71.8 & $\mathrm{I}-\mathrm{ACT}$ & $\mathrm{S} / \mathrm{l}$ & Inactive & 12 months \\
\hline Levin, Pistorello, Seeley, \& Hayes, 2014 & College students & 76 & 18.4 & 54 & $\mathrm{I}-\mathrm{ACT}$ & $U$ & Inactive & 3 weeks \\
\hline Ljótsson et al., 2010 & IBS patients & 85 & 34.6 & 84.7 & $\mathrm{I}-\mathrm{E}$ and $\mathrm{MBI}$ & $\mathrm{S} / \mathrm{l}$ & Active & 3 months \\
\hline Ljótsson, Andersson, et al., 2011 & IBS patients & 61 & 34.9 & 74 & $\mathrm{I}-\mathrm{E}$ and $\mathrm{MBI}$ & $\mathrm{S} / \mathrm{l}$ & Inactive & 12 months \\
\hline Ljótsson, Hedman, et al., 2011 & IBS patients & 195 & 38.9 & 79 & $\mathrm{I}-\mathrm{E}$ and $\mathrm{MBI}$ & $\mathrm{S} / \mathrm{l}$ & Intervention & 6 months \\
\hline Ly et al., 2014 & Adults diagnosed with major depression & 81 & 36.1 & 70 & S-MBCT & $\mathrm{T}$ & Intervention & 6 months \\
\hline Pots et al., 2015 & Adults experiencing depressive symptoms & 236 & 46.8 & 75.8 & $\mathrm{I}-\mathrm{ACT}$ & $\mathrm{S} / \mathrm{l}$ & Active; inactive & 6 and 12 months \\
\hline Shapira \& Mongrain, 2010 & Adults from nonclinical population & 1,002 & 34 & 81.5 & $\mathrm{I}-\mathrm{CMT}$ & $\mathrm{U}$ & Active; intervention & 1,3 , and 6 months \\
\hline Trompetter, Bohlmeijer, Veehof, \& Schreurs, 2015 & Adults experiencing chronic pain & 238 & 52.8 & 76 & $\mathrm{I}-\mathrm{ACT}$ & $\mathrm{S} / \mathrm{l}$ & Active; inactive & 3 months \\
\hline Weineland, Arvidsson, Kakoulidis, \& Dahl, 2012 & Bariatric surgery patients & 39 & 43.1 & 89.7 & $\mathrm{I}-\mathrm{ACT}$ & $\mathrm{S} / \mathrm{l}$ & Intervention & None \\
\hline
\end{tabular}

Note. NS = not stated; C-CBASP = computer-based cognitive-behavioral analysis system of psychotherapy; I-ACT = Internet-delivered acceptance and commitment therapy; I-BA = Internet-delivered behavioral activation; I-MBCT = Internet-delivered mindfulness-based cognitive therapy; I-ABBT = Internet-delivered acceptance-based behavior therapy; I-CFT =

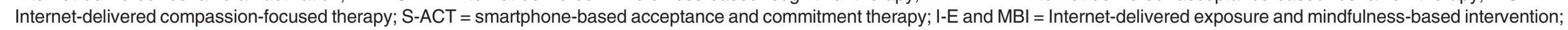
$\mathrm{S}-\mathrm{MBCT}=$ smartphone-based mindfulness-based cognitive therapy; I-CMT = Internet-delivered compassionate mind training; $\mathrm{U}=$ universal; $\mathrm{S} / \mathrm{I}=$ selective/indicated; $\mathrm{T}=$ treatment. 
of which ranged from 4 to 12 weeks. Carlbring et al. (2013) and Kivi et al. (2014) tested an 8-week guided Internet-delivered intervention combining components of behavioral activation and ACT. One study evaluated an 8-week guided MBCT program administered via smartphone application (Ly et al., 2014), while another examined an unguided Internetdelivered MBCT intervention (Dowd et al., 2015). An off-line computer-based CBASP treatment was tested by Braithwaite and Fincham (2007) and Cukrowicz and Joiner (2007). Three studies evaluated a 10-week guided Internet-delivered exposure and mindfulnessbased intervention "inspired by the third wave of CBT” (Ljótsson, Andersson, et al., 2011, p. 4; Ljótsson et al., 2010; Ljótsson, Hedman, et al., 2011). Glick and Orsillo (2015) investigated the efficacy of Internet-delivered acceptance-based behavior therapy. CFT and CMT informed the remaining unguided Internet-delivered treatments (Kelly \& Carter, 2015; Shapira \& Mongrain, 2010).

\section{Comparators}

Eight studies compared third-wave eHealth treatments with inactive (i.e., no treatment or waitlist) control conditions. Active control conditions featured in eight studies: Internet-based expressive writing, online discussion forum, early memory writing exercise, and off-line computer-based psychoeducation. Third-wave eHealth was compared with a comparison intervention in 11 studies. The comparison interventions included face-to-face ACT, treatment as usual, Internet-delivered cognitive-behavior therapy, pain management psychoeducation, stress management, time management, behavioral strategies, and optimism interventions. In addition, a smartphone application based on behavioral activation was used as a comparison intervention as was an off-line computer-based prevention and relationship enhancement program (ePREP).

\section{Outcome Measures}

Seventeen studies included a self-report measure of depressive symptoms. Measures of depressive symptoms included the Beck Depression Inventory (Beck, Steer, \& Carbin, 1988; Beck, Steer, \& Brown, 1996), Center for Epidemiologic Studies Depression Scale (Radloff, 1977), depression scale of the Depression Anxiety and Stress Scales (Lovibond \& Lovibond, 1995), Montgomery Asberg Depression Rating Scale (Svanborg \& Asberg, 1994), and the depression subscale of the Hospital Anxiety and Depression Scale (Zigmond \& Snaith, 1983). Anxiety symptomology was assessed in 13 studies; measures included the Beck Anxiety Inventory (Beck, Epstein, Brown, \& Steer, 1988), anxiety scale of the Depression Anxiety and Stress Scales, State-Trait Anxiety Inventory (Spielberger, Gorsuch, Lushene, Vagg, \& Jacobs,
1983), and the anxiety subscale of the Hospital Anxiety and Depression Scale. Quality of life was assessed in nine studies. Measures of quality of life included the Quality of Life Inventory (Frisch, Cornell, Villanueva, \& Retzlaff, 1992), Short Form Health Survey (SF-8; Ware, Kosinski, Dewey, \& Gandek, 2001), Irritable Bowel Syndrome Quality of Life Instrument (Patrick, Drossman, Frederick, DiCesare, \& Puder, 1998), and the World Health Organization Quality of Life-BREF instrument (WHOQOL Group, 1998).

\section{Secondary Outcomes}

Eleven studies included self-report measures of the acceptability of the third-wave eHealth treatments. Participants rated the treatments as satisfactory (Trompetter et al., 2015; Lappalainen et al., 2014, 2015), enjoyable (Glick \& Orsillo, 2015), useful (Kristjánsdóttir et al., 2013), and helpful (Cukrowicz \& Joiner, 2007), as measured by the Client Satisfaction Questionnaire (Attkisson \& Zwick, 1982), visual analogue scales, and brief questionnaires, respectively. Four studies administered the Credibility/Expectancy Questionnaire (Borkovec \& Nau, 1972; Devilly \& Borkovec, 2000) and ratings indicated that participants perceived the third-wave eHealth treatments as credible (Kelly \& Carter, 2015; Ljótsson et al., 2010; Ljótsson, Hedman et al., 2011; Ly et al., 2014). Participants rated the Internet-delivered intervention administered by Levin, Pistorello, Seeley, and Hayes (2014) within the top $10 \%$ of scores on the System Usability Scale (Tullis \& Albert, 2008).

\section{RISK OF BIAS WITHIN STUDIES}

The results of the risk of bias assessment of included studies are presented in Table 2. Thirteen studies reported adequate generation of a randomized sequence; 8 provided insufficient information to judge risk of bias in this domain. Adequate concealment of the allocation sequence was reported in 14 studies. Seven studies provided insufficient detail to permit judgment of "low risk" or "high risk" of selection bias due to inadequate concealment of the allocation sequence. Blinding of study participants and personnel to treatment allocation is possible in eHealth trials featuring active control conditions or comparison interventions (Tait, Spijkerman, \& Riper, 2013). In accordance, 7 studies were judged to be at low risk of performance bias, while a high risk of bias was present in the remaining 14 studies due to participants' knowledge of treatment allocation. Consequently, there was a low risk that the selfreport outcome measures in the aforementioned 7 studies were influenced by lack of blinding. Seventeen of the included studies featured intention-to-treat analyses, 12 of which had low rates of missing 
Table 2

Risk of Bias Within Studies

\begin{tabular}{|c|c|c|c|c|c|c|c|}
\hline \multirow[b]{2}{*}{ Study } & \multicolumn{2}{|l|}{ Selection bias } & \multirow{2}{*}{$\begin{array}{l}\text { Performance bias } \\
\text { Blinding of participants } \\
\text { and personnel }\end{array}$} & \multirow{2}{*}{$\begin{array}{l}\text { Detection bias } \\
\text { Blinding of outcome } \\
\text { assessment }\end{array}$} & \multirow{2}{*}{$\begin{array}{l}\frac{\text { Attrition bias }}{\text { Incomplete }} \\
\text { outcome data }\end{array}$} & \multirow{2}{*}{$\begin{array}{l}\text { Reporting bias } \\
\text { Selective } \\
\text { reporting }\end{array}$} & \multirow[b]{2}{*}{$\begin{array}{l}\text { Other } \\
\text { bias }\end{array}$} \\
\hline & $\begin{array}{l}\text { Random sequence } \\
\text { generation }\end{array}$ & $\begin{array}{l}\text { Allocation } \\
\text { concealment }\end{array}$ & & & & & \\
\hline Braithwaite \& Fincham, 2007 & Unclear & Unclear & Low & Low & High & Unclear & Low \\
\hline Buhrman et al., 2013 & Low & Low & High & High & Unclear & Low & Low \\
\hline Carlbring et al., 2013 & Low & Low & High & High & Low & Unclear & Low \\
\hline Cukrowicz \& Joiner, 2007 & Unclear & Unclear & Low & Low & High & Unclear & Low \\
\hline Dowd et al., 2015 & Low & Low & High & High & High & Unclear & Low \\
\hline Glick \& Orsillo, 2015 & Unclear & Unclear & Low & Low & High & Unclear & Low \\
\hline Hesser et al., 2012 & Low & Low & High & High & Low & Unclear & Low \\
\hline Kelly \& Carter, 2015 & Low & Unclear & High & High & Low & Unclear & Low \\
\hline Kivi et al., 2014 & Unclear & Low & High & High & High & Unclear & Low \\
\hline Kristjánsdóttir et al., 2013 & Low & Low & High & High & High & Low & Low \\
\hline Lappalainen et al., 2014 & Unclear & Unclear & Low & Low & Low & Unclear & Low \\
\hline Lappalainen et al., 2015 & Unclear & Low & High & High & Low & Unclear & Low \\
\hline Levin et al., 2014 & Unclear & Unclear & High & High & Low & Unclear & Low \\
\hline Ljótsson et al., 2010 & Low & Low & High & High & Low & Unclear & Low \\
\hline Ljótsson, Andersson, et al., 2011 & Low & Low & High & High & Low & Low & Low \\
\hline Ljótsson, Hedman, et al., 2011 & Low & Low & Low & Low & Low & Low & Low \\
\hline Ly et al., 2014 & Low & Low & Low & Low & Low & Low & Low \\
\hline Pots et al., 2015 & Low & Low & High & High & Low & Low & Low \\
\hline Shapira \& Mongrain, 2010 & Low & Low & Low & Low & High & Unclear & Low \\
\hline Trompetter et al., 2015 & Low & Low & High & High & High & Low & Low \\
\hline Weineland et al., 2012 & Unclear & Unclear & High & High & Low & Unclear & Low \\
\hline
\end{tabular}


outcome data and were judged to be at low risk of attrition bias. Registered protocols that prespecified the reported study outcomes were found for 7 studies; protocols could not be found to assess risk of reporting bias in the remaining 14 studies. All of the included studies were deemed to be free of other sources of bias, such as baseline imbalances in variables strongly related to outcome measures.

\section{SYNTHESES OF RESULTS}

The meta-analyses of included studies are summarized in Table 3.

\section{Depression}

Six studies that compared third-wave eHealth treatments with inactive control conditions reported depression outcomes. A random effects meta-analysis combining the results of these studies revealed that third-wave eHealth had a medium statistically significant effect relative to inactive control $(g=0.52$, $S E=0.13,95 \%$ CI $[0.26,0.77], p<.01)$. Heterogeneity analyses indicated the presence of a low degree of heterogeneity between studies $(Q=9.75$, 95\% CI [-1.17, 20.67], $\left.I^{2}=48.74\right)$. Excluding two studies (Carlbring et al., 2013; Trompetter et al., 2015) with high standardized residuals from the analysis removed this heterogeneity $(Q=1.99,95 \%$ CI $\left.[-4.87,8.85], I^{2}=0.00\right)$ and did not affect the treatment effect $(g=0.52, S E=0.11,95 \%$ CI [0.29, $0.74], p<.01)$.

A random effects meta-analysis of eight studies that compared third-wave eHealth treatments with active control conditions showed that third-wave eHealth was superior in improving depression outcomes with a small ES $(g=0.29, S E=0.08,95 \%$ CI $[0.14,0.44], p<.01)$. The analysis featured a low degree of heterogeneity $(Q=10.44,95 \%$ CI $[-4.47$, 25.35], $I^{2}=32.97$ ).

Nine studies investigated the efficacy of third-wave eHealth treatments relative to comparison interven- tions in improving depression outcomes. There was no statistically significant difference between the treatments in depression outcomes $(g=-0.02, S E=$ $0.08,95 \%$ CI $[-0.18,0.15], p=.83 ; Q=10.41,95 \%$ CI $\left.[-6.95,27.77], I^{2}=23.14\right)$.

\section{Anxiety}

Four studies that compared third-wave eHealth treatments with inactive control conditions reported anxiety outcomes. Third-wave eHealth was significantly more efficacious in improving anxiety outcomes with a small ES $(g=0.32, S E=0.12,95 \%$ CI $[0.09,0.56], p=.01)$. Heterogeneity analyses indicated the presence of a low degree of variability between studies $(Q=5.02,95 \%$ CI $[-2.15,12.19]$, $\left.I^{2}=40.23\right)$.

A random effects meta-analysis of six studies that compared third-wave eHealth treatments with active control conditions showed that third-wave eHealth was superior in improving anxiety outcomes with a small ES $(g=0.31, S E=0.12,95 \%$ CI $[0.07,0.54], p=$ $.01)$. The analysis featured a moderate degree of heterogeneity $\left(Q=10.98,95 \% \mathrm{CI}[-0.02,21.98], I^{2}=\right.$ 54.47). Excluding two studies (Hesser et al., 2012; Trompetter et al., 2015) with high standardized residuals from the analysis removed this heterogeneity $\left(Q=2.71,95 \%\right.$ CI $\left.[-4.17,9.59], I^{2}=0.00\right)$ and did not affect the small treatment effect $(g=0.35, S E=$ $0.10,95 \%$ CI $[0.16,0.54], p<.01)$.

Seven studies investigated the efficacy of thirdwave eHealth treatments relative to comparison interventions in improving anxiety outcomes. There was no difference between the treatments in anxiety outcomes $(g=0.00, S E=0.08,95 \%$ CI $[-0.16$, $0.17], p=.97 ; Q=3.78,95 \%$ CI $[-16.25,23.81]$, $\left.I^{2}=0.00\right)$.

Quality of Life

Three studies investigated the efficacy of thirdwave eHealth treatments relative to inactive control conditions in improving quality of life. Third-wave

Table 3

Meta-Analyses of the Effects of Third-Wave eHealth Relative to Comparators on Mental Health Outcomes and Participant Attrition

\begin{tabular}{llllll}
\hline Outcome & Comparator & $N$ & ES & $95 \% \mathrm{Cl}$ & $p$ \\
\hline Depression & Inactive control & 6 & $g=0.52$ & {$[0.26,0.77]$} & {$[0.14,0.44]$} \\
Depression & Active control & 8 & $g=0.29$ & .01 \\
Depression & Comparison intervention & 9 & $g=-0.02$ & {$[-0.18,0.15]$} & .83 \\
Anxiety & Inactive control & 4 & $g=0.32$ & {$[0.09,0.56]$} & .01 \\
Anxiety & Active control & 6 & $g=0.31$ & {$[0.07,0.54]$} & .01 \\
Anxiety & Comparison intervention & 7 & $g=0.00$ & {$[-0.16,0.17]$} & .97 \\
Quality of life & Inactive control & 3 & $g=0.46$ & {$[0.00,0.92]$} & .05 \\
Quality of life & Active control & 3 & $g=0.31$ & {$[-0.31,0.93]$} & .33 \\
Quality of life & Comparison intervention & 4 & $g=0.22$ & {$[-0.21,0.65]$} & .31 \\
Participant attrition & Inactive control & 8 & $O R=1.24$ & {$[0.82,1.86]$} & .30 \\
Participant attrition & Active control & 7 & $O R=1.03$ & {$[0.63,1.66]$} & .92 \\
Participant attrition & Comparison intervention & 10 & $O R=1.03$ & {$[0.79,1.33]$} & .84 \\
\hline
\end{tabular}


eHealth was superior in improving quality of life with a small $\mathrm{ES}(g=0.46, S E=0.23,95 \%$ CI [0.00, $0.92], p=.05)$. Heterogeneity analyses indicated the presence of a moderate degree of variability between studies $(Q=5.71,95 \%$ CI $[-0.25,11.67]$, $I^{2}=64.96$ ). Excluding a study (Carlbring et al., 2013) with a high standardized residual from the analysis removed this heterogeneity $(Q=0.18$, $95 \%$ CI $\left.[-0.64,1.00], I^{2}=0.00\right)$, but increased the estimated ES $(g=0.68, S E=0.17,95 \%$ CI $[0.34$, 1.03 ], $p<.01)$.

A random effects meta-analysis of three studies that compared third-wave eHealth treatments with active control conditions in improving quality of life revealed no statistically significant difference between conditions $(g=0.31, S E=0.32,95 \%$ CI $[-0.31$, $0.93], p=.33 ; Q=11.33,95 \% \mathrm{CI}[2.71,19.95], I^{2}=$ 82.34). The exclusion of a study (Ljótsson et al., 2010) with a high standardized residual removed this heterogeneity $\left(Q=0.31,95 \%\right.$ CI $[-0.79,1.41], I^{2}=$ $0.00)$ and the difference between conditions remained nonsignificant.

Four studies investigated the efficacy of thirdwave eHealth treatments relative to comparison interventions in improving quality of life. There was no statistically significant difference between the treatments in quality of life $(g=0.22, S E=0.22$, $95 \%$ CI $[-0.21,0.65], p=.31)$. The analysis featured a high degree of heterogeneity $(Q=12.12$, $95 \%$ CI $\left.[2.89,21.35], I^{2}=75.24\right)$. Excluding two studies (Hesser et al., 2012; Weineland et al., 2012) with high standardized residuals did not remove this heterogeneity $(Q=5.07,95 \%$ CI $[0.80,9.34]$, $\left.I^{2}=80.27\right)$.

\section{PARTICIPANT ATTRITION}

Third-Wave eHealth Compared With Inactive Control

Attrition rates were extracted from the eight studies that compared third-wave eHealth treatments with inactive control conditions and combined in a random effects meta-analysis. The analysis revealed that participant dropout did not significantly differ between conditions $(O R=1.24,95 \%$ CI $[0.82$, $1.86], p=.30)$. Heterogeneity analyses indicated no significant variability between studies $(Q=5.68$, $95 \%$ CI $\left.[-14.57,25.93], I^{2}=0.00\right)$.

Third-Wave eHealth Compared With Active Control Seven of the studies that compared third-wave eHealth with active control conditions provided participant attrition rates. Participant attrition did not significantly differ between conditions $(O R=$ $1.03,95 \%$ CI $[0.63,1.66], p=.92)$. A low degree of heterogeneity was present in the analysis $(Q=9.97$, $95 \%$ CI $\left.[-2.94,22.88], I^{2}=39.82\right)$.
Third-Wave eHealth Compared With Comparison Intervention

A random effects meta-analysis of 10 studies that provided attrition rates revealed no statistically significant difference in participant dropout between treatments $(O R=1.03,95 \%$ CI $[0.79,1.33], p=$ .84). Heterogeneity analyses indicated no statistically significant variability between studies $(Q=7.86$, $95 \%$ CI $\left.[-17.95,33.67], I^{2}=0.00\right)$.

\section{SUBGROUP ANALYSES}

Subgroup analyses were conducted to investigate differences in ES between universal, selective/ indicated, and treatment interventions. As shown in Table 4, no statistically significant differences were found between the subgroups on depression, anxiety, or quality-of-life outcomes.

\section{RISK OF BIAS ACROSS STUDIES}

Funnel plots were produced to explore potential publication bias on summary effect measures. A funnel plot of studies that compared third-wave eHealth with active control conditions on depression outcomes showed some asymmetry. Correction for potential publication bias using Duval and Tweedie's (2000) Trim and fill procedure reduced the ES from $g=0.29(95 \% \mathrm{CI}[0.14,0.44])$ to $g=0.16(95 \% \mathrm{CI}$ $[0.00,0.32])$. No evidence of publication bias was detected among studies that compared third-wave eHealth with inactive control conditions or comparison interventions on depression outcomes. Slight asymmetry in a funnel plot of studies that compared third-wave eHealth with inactive control conditions on anxiety outcomes was corrected using Trim and fill: the summary effect measure was adjusted from $g=0.32(95 \%$ CI $[0.09,0.56])$ to $g=0.25(95 \%$ CI $[0.03,0.47])$. There was no indication of publication bias among studies that compared third-wave eHealth with active control conditions or comparison interventions on anxiety outcomes. Similarly, no

Table 4

Subgroup Analyses of the Overall Effect Size of Third-Wave eHealth on Mental Health Outcomes

\begin{tabular}{llllll}
\hline & $N$ & $g$ & $95 \% \mathrm{Cl}$ & $Q$ & $p$ \\
\hline $\begin{array}{l}\text { Depression } \\
\quad \text { Universal }\end{array}$ & 4 & 0.13 & {$[-0.22,0.48]$} & & \\
$\quad$ Selective/indicated & 9 & 0.30 & {$[0.06,0.55]$} & & \\
$\quad$ Treatment & 4 & 0.45 & {$[0.00,0.90]$} & & \\
Anxiety & & & & 1.09 & .58 \\
$\quad$ Universal & 4 & 0.29 & {$[0.03,0.55]$} & & \\
$\quad$ Selective/indicated & 6 & 0.11 & {$[-0.09,0.32]$} & & \\
$\quad$ Treatment & 3 & 0.17 & {$[-0.23,0.56]$} & & \\
$\quad \begin{array}{l}\text { Quality of life } \\
\quad \text { Selective/indicated }\end{array}$ & 7 & 0.48 & {$[0.21,0.75]$} & & \\
$\quad$ Treatment & 2 & -0.02 & {$[-0.51,0.47]$} & & \\
\hline
\end{tabular}


evidence of publication bias was detected among studies that estimated the effects of third-wave eHealth relative to inactive or active control conditions on quality of life. There was, however, slight asymmetry in a funnel plot of studies that compared third-wave eHealth with comparison interventions: Trim and fill adjusted the nonsignificant effect of third-wave eHealth from $g=0.22(95 \%$ CI $[-0.21$, $0.65])$ to $g=0.09(95 \%$ CI $[-0.35,0.53])$.

\section{META-REGRESSION ANALYSES}

Meta-regression analyses were conducted to investigate associations between domains of bias and ES. Blinding of participants, personnel, and outcome assessors was significantly associated with depression outcomes, such that studies with an unclear or high risk of bias were associated with higher ES $(b=$ $0.39, Z=2.34,95 \%$ CI [0.06, 0.71], $p=0.02$ ). Risk of selection bias, attrition bias, or reporting bias did not significantly moderate depression outcomes $(p>.05)$. The domains of bias were not significantly associated with ES for anxiety or quality-of-life outcomes $(p>.05)$. Meta-regression analyses were also conducted to investigate the potential moderating effects of intervention delivery mode (i.e., Internet delivered, off-line computer based, or smartphone based), number of intervention sessions, and therapist guidance (i.e., with or without) on depression, anxiety, and quality-of-life outcomes. Intervention delivery mode, number of intervention sessions, or therapist guidance did not significantly moderate the mental health outcomes $(p>.05)$. In addition, study population (i.e., nonclinical, somatic illnesses, or psychological illnesses) was not significantly associated with ES for depression, anxiety, or quality-of-life outcomes $(p>.05)$.

\section{Discussion}

This systematic review and meta-analysis aimed to determine the efficacy of third-wave eHealth treatments in improving mental health outcomes including anxiety, depression, and quality of life. In accordance, 21 randomized controlled trials were included in the review. Findings indicated that third-wave eHealth significantly outperformed inactive control conditions in improving depression, anxiety, and quality-of-life outcomes at posttreatment, resulting in small to medium ESs. Third-wave eHealth treatments were also found to be significantly more efficacious than active control conditions in alleviating depression and anxiety with small ESs. No statistically significant differences were found relative to comparison interventions.

For depression outcomes, third-wave eHealth treatments produced smaller EFs relative to active control conditions $(g=0.29)$ than inactive control conditions $(g=0.52)$. This finding suggests that more stringent study designs yield smaller EFs and concurs with previous meta-analyses (e.g., Öst, 2008). Moreover, this finding highlights the moderating effects of studies' type of comparison condition in meta-analyses. Interestingly, the small statistically significant effect of third-wave eHealth compared with inactive control conditions on anxiety outcomes ( $g=0.32$ ) was analogous to that found relative to active control conditions $(g=0.31)$. This finding is also demonstrated in the individual interventional trials synthesised in these analyses that included both inactive and active control conditions. For example, Pots et al. (2015) found that an Internetdelivered ACT intervention yielded a small statistically significant effect on anxiety outcomes relative to both inactive $(g=0.49)$ and active control conditions $(g=0.41)$. Conversely, third-wave eHealth yielded a small statistically significant effect on quality of life relative to inactive controls $(g=0.46)$, but no statistically significant differences were found relative to active controls or comparison interventions. Given the emphasis that third-wave therapies place on quality of life (Forsyth \& Sheppard, 2009), this finding is disappointing but such inferences should be tempered by the small number of studies synthesised in these meta-analyses.

In line with these findings, a recent meta-analytic review by Spijkerman et al. (2016) found that online mindfulness-based interventions produced small to medium statistically significant benefits on depression, anxiety, and well-being outcomes at posttreatment relative to control conditions. In addition, this recent meta-analytic review also found that ESs did not significantly vary as a function of study population (i.e., nonclinical, somatic illnesses, or psychological illnesses). Spijkerman et al. (2016), however, were unable to conduct subgroup analyses based on studies' type of comparison condition. Similarly, a systematic review by Cavanagh et al. (2014) found that mindfulness- and acceptance-based self-help interventions had small statistically significant benefits on anxiety and depression outcomes relative to comparison conditions. Although Cavanagh et al. (2014) included trials of books/workbooks, audio recordings, and Internet-delivered interventions, four of which are featured in this review, the current findings support and expand upon their conclusion that contextual or third-wave treatments can be delivered efficaciously in various formats. Conversely, the estimated effects of third-wave eHealth treatments in the current review are lower than those of third-wave therapies delivered in individual and group face-to-face format (Öst, 2008). Thus, the efficacy of third-wave treatments in their traditional format may be attenuated when delivered via eHealth. However, this is a tentative 
inference, as the overall treatment effect in the aforementioned review of third-wave face-to-face therapies was an amalgam of a diverse range of somatic and psychological outcomes.

Perhaps as important as the efficacy of third-wave eHealth treatments in improving mental health outcomes is their acceptability to participants. In accordance, a narrative synthesis of participant evaluation outcomes in the current review provided preliminary support for the acceptability of these treatments. Specifically, the third-wave eHealth interventions were rated as satisfactory, helpful, useful, enjoyable, and credible. Furthermore, previous research (e.g., Kenardy, McCafferty, \& Rosa, 2003; Mitchell \& Gordon, 2007) has investigated participant attrition as a proxy measure of acceptability and attitudes toward eHealth treatments (Davies et al., 2014). Meta-analyses of studies' attrition rates in the current review revealed no statistically significant differences in the odds of participant dropout in treatment groups relative to inactive, active control conditions or comparison interventions. This finding indicates that the participants perceived the third-wave eHealth treatments to be as tolerable as the comparison conditions. However, Eysenbach (2005) suggests that eHealth trials are often characterized by extremely high dropout rates-the so-called law of attrition. In this review, $23 \%$ of participants randomized to thirdwave eHealth treatments did not complete posttreatment assessments. Thus, there is a risk that the summary effect measures in this review may be exaggerated due to the inclusion of four trials that restricted analyses to study completers.

The included studies varied in their risk of biasing this review's estimates of the efficacy of third-wave eHealth treatments. The majority of studies were at low risk of selection bias due to adequate random sequence generation $(62 \%)$ and allocation concealment $(67 \%)$. In contrast, a large proportion of included studies were at high risk of performance $(67 \%)$ and detection bias (67\%), mainly due to the preponderance of inactive control conditions and self-report measures, respectively. This potential performance and detection bias was significantly associated with higher ES for depression outcomes. An unclear risk of reporting bias was common, however, as study protocols could not be found for $67 \%$ of trials. Consequently, these potential sources of bias in included studies should be carefully considered when interpreting the results of this review.

In addition to risk of bias in included studies, limitations in the review process should be taken into consideration. First, this review's search strategy was restricted to randomized controlled trials published in English-language peer-reviewed journals-no non-English language or gray literature was sought. Thus, language or publication biases may have affected the results of this review, as studies with positive findings are more likely to be submitted for publication and published in English-language journals (Sterne, Egger, \& Moher, 2011). Given that smaller studies have greater variability in their estimates of a treatment's effect, a meta-analysis in which the estimates of smaller studies are disproportionally greater than the population ES can be indicative of publication bias (Field \& Gillett, 2010). Correcting for such potential publication bias in this review reduced two statistically significant ES estimates. Second, the meta-analyses in this review were restricted to data collected at posttreatment assessments. Therefore, the analyses do not elucidate whether the beneficial effects of third-wave eHealth are sustained over an extended period of time. Furthermore, this systematic review featured an extensive search of electronic bibliographic databases (PubMed, PsycINFO, Web of Science, and CENTRAL), relevant review articles, and reference lists of included studies, but yielded a relatively small number of studies for inclusion of the meta-analyses. However, Valentine, Pigott, and Rothstein (2010) argue that even when few studies are found to meet a review's inclusion criteria, meta-analysis is more likely to yield a valid conclusion than all other synthesis techniques (e.g., narrative summary), as they are based on less transparent processes and on less defensible assumptions. A third limitation of this review is that many third-wave approaches including DBT, FAP, IBCT, MCT, and schema therapy had no eligible studies. Moreover, previous literature varies in its categorization of third-wave approaches (Churchill et al., 2013) and some (e.g., Hofmann, Sawyer, \& Fang, 2010) even argue that these treatments do not warrant a separate classification in the family of cognitive-behavioral therapies. Despite these differing perspectives, this review featured the most comprehensive categorization of third-wave approaches to date. A final limitation concerns the diversity in populations sampled and outcome measures utilized by studies included in the meta-analyses. However, random effects models were employed to account for heterogeneity in study populations. Furthermore, this diversity is likely an accurate representation of the scope of third-wave eHealth treatments.

With these limitations in mind, the findings of this review have implications for health care providers and their clients. The findings suggest that thirdwave therapies can be delivered efficaciously via modern information technology and electronic communication resources to a range of community 
and clinical populations, producing small to medium statistically significant improvements in mental health outcomes including anxiety, depression, and quality of life. Furthermore, subgroup analyses revealed no statistically significant differences in ES as a function of type of intervention: universal, selective/indicated, and treatment. Given that thirdwave approaches focus on promoting broad and flexible repertoires and neutralizing or reversing the ubiquitous psychological processes theorized to be responsible for much human suffering, third-wave eHealth may hold promise for improving access to mental health services (Clark, 2011) and existing stepped care approaches (Firth, Barkham, \& Kellett, 2015). However, further research, particularly studies investigating third-wave universal and treatment eHealth interventions, is necessary to substantiate such assertions. In addition, the generalizability of these treatments is limited to individuals who have access to Internet technologies or mobile devices. Currently, only $49.5 \%$ of the world's population are Internet users (Internet World Stats, 2016) and inequalities exist in the utilization of information and communication technologies (Mendonça, Crespo, \& Simões, 2015). Indeed, individuals with low socioeconomic status are more likely to experience psychological problems (Hudson, 2005), but less likely to have access to the Internet (Pew Research Center, 2012). Consequently, the reach of eHealth treatments is confined by the digital divide. Moreover, the findings of this review should not be generalized to children or adolescents, as they were not represented in the included studies.

The paucity of research into the efficacy of eHealth treatments for children and adolescents is reflected in recent systematic reviews (e.g., Arnberg, Linton, Hultcrantz, Heintz, \& Jonsson, 2014; Stephens \& Allen, 2013). In accordance, future research should investigate the efficacy of thirdwave eHealth for children and adolescents in adequately powered randomized controlled trials that address the methodological limitations highlighted in this review. The findings from a recent open pilot trial of multimodal DBT for school refusal show promise for such treatments (Chu, Rizvi, Zendegui, \& Bonavitacola, 2015). In addition, future research should extend the work of Lappalainen et al. (2014) by investigating whether third-wave eHealth treatments are noninferior to existing empirically supported therapies.

In conclusion, the findings of this systematic review and meta-analysis suggest that third-wave eHealth treatments are efficacious in improving mental health outcomes including anxiety, depression, and quality of life, but not more so than comparison interventions. Preliminary evidence from indices of participant evaluation and attrition rates supports the acceptability of these treatments. However, limitations in the included studies and review process should be carefully considered when interpreting these findings. Future research should focus on evaluating the efficacy of third-wave eHealth for underrepresented cohorts including children and adolescents and investigating whether these treatments are noninferior to empirically supported therapies. These applications have the potential to improve the dissemination of the third wave of behavioral and cognitive therapy and alleviate the suffering of those who currently lack access to it.

\section{Conflict of Interest Statement}

The authors declare that there are no conflicts of interest.

\section{Appendix A. Supplementary Data}

Supplementary data to this article can be found online at http://dx.doi.org/10.1016/j.beth.2017.07. 007.

\section{References '}

Andersson, G., \& Titov, N. (2014). Advantages and limitations of internet-based interventions for common mental disorders. World Psychiatry, 13(1), 4-11. http://dx.doi.org/10.1002/ wps.20083

Arnberg, F. K., Linton, S. J., Hultcrantz, M., Heintz, E., \& Jonsson, U. (2014). Internet-delivered psychological treatments for mood and anxiety disorders: A systematic review of their efficacy, safety, and cost-effectiveness. PLOS ONE, 9(5), e98118. http://dx.doi.org/10.1371/journal.pone.0098118

A-Tjak, J. G., Davis, M. L., Morina, N., Powers, M. B., Smits, J. A., \& Emmelkamp, P. M. (2015). A meta-analysis of the efficacy of acceptance and commitment therapy for clinically relevant mental and physical health problems. Psychotherapy and Psychosomatics, 84(1), 30-36. http://dx.doi.org/10.1159/ 000365764

Attkisson, C. C., \& Zwick, R. (1982). The Client Satisfaction Questionnaire: Psychometric properties and correlations with service utilization and psychotherapy outcome. Evaluation and Program Planning, 5(3), 233-237.

Beck, A. T., Epstein, N., Brown, G., \& Steer, R. (1988). An inventory for measuring clinical anxiety: Psychometric properties. Journal of Consulting and Clinical Psychology, 56(6), 893-897. http://dx.doi.org/10.1037/0022-006X.56.6.893

Beck, A. T., Steer, R. A., \& Brown, G. K. (1996). Manual for the Beck Depression Inventory-II. San Antonio, TX: Psychological Corporation.

Beck, A. T., Steer, R. A., \& Carbin, M. G. (1988). Psychometric properties of the Beck Depression Inventory: Twenty-five years of evaluation. Clinical Psychology Review, 8(1), 77-100. http://dx.doi.org/10.1016/0272-7358(88)90050-5

Bluett, E. J., Homan, K. J., Morrison, K. L., Levin, M. E., \& Twohig, M. P. (2014). Acceptance and commitment therapy for anxiety and OCD spectrum disorders: An empirical review. Journal of Anxiety Disorders, 28, 612-624. http://dx.doi.org/ 10.1016/j.janxdis.2014.06.008

\footnotetext{
${ }^{1}$ References for studies included in meta-analyses are presented in an online supplementary file.
} 
Blundell, M. (2014). Understanding and synthesizing my numerical data. In A. Boland, M. G. Cherry, \& R. Dickson (Eds.), Doing a systematic review (pp. 99-123). London, UK: Sage.

Borenstein, M., Hedges, L. V., Higgins, J. P., \& Rothstein, H. R. (2009). Effect sizes based on means. In Introduction to meta-analysis (pp. 21-32). Chichester, UK: Wiley.

Borkovec, T., \& Nau, S. (1972). Credibility of analogue therapy rationales. Journal of Behavior Therapy and Experimental Psychiatry, 3(4), 257-260. http://dx.doi.org/10.1016/00057916(72)90045-6

Braithwaite, S. R., \& Fincham, F. D. (2007). ePREP: Computer based prevention of relationship dysfunction, depression and anxiety. Journal of Social and Clinical Psychology, 26(5), 609-622. http://dx.doi.org/10.1521/jscp.2007.26.5.609

Buhrman, M., Skoglund, A., Husell, J., Bergström, K., Gordh, T., Hursti, T., . . . Andersson, G. (2013). Guided internetdelivered acceptance and commitment therapy for chronic pain patients: A randomized controlled trial. Behaviour Research and Therapy, 51, 307-315. http://dx.doi.org/10. 1016/j.brat.2013.02.010

Carlbring, P., Hagglund, M., Luthstrom, A., Dahlin, M., Kadowaki, A., Vernmark, K., \& Andersson, G. (2013). Internet-based behavioral activation and acceptance-based treatment for depression: A randomized controlled trial. Journal of Affective Disorders, 148, 331-337. http://dx.doi.org/10. 1016/j.jad.2012.12.020

Cavanagh, K., Strauss, C., Forder, L., \& Jones, F. (2014). Can mindfulness and acceptance be learnt by self-help?: A systematic review and meta-analysis of mindfulness and acceptance-based self-help interventions. Clinical Psychology Review, 34(2), 118-129. http://dx.doi.org/10.1016/j.cpr.2014.01.001

Chiesa, A., \& Serretti, A. (2011). Mindfulness based cognitive therapy for psychiatric disorders: A systematic review and meta-analysis. Psychiatry Research, 187, 441-453. http://dx. doi.org/10.1016/j.psychres.2010.08.011

Chu, B. C., Rizvi, S. L., Zendegui, E. A., \& Bonavitacola, L. (2015). Dialectical behavior therapy for school refusal: Treatment development and incorporation of web-based coaching. Cognitive and Behavioral Practice, 22(3), 317-330. http://dx. doi.org/10.1016/j.cbpra.2014.08.002

Churchill, R., Moore, T. H., Furukawa, T. A., Caldwell, D. M., Davies, P., Jones, H., . . . Hunot, V. (2013). "Third wave" cognitive and behavioural therapies versus treatment as usual for depression. Cochrane Database of Systematic Reviews, 10. http://dx.doi.org/10.1002/14651858.CD008705.pub2

Clark, D. (2011). Implementing NICE guidelines for the psychological treatment of depression and anxiety disorders: The IAPT experience. International Review of Psychiatry, 23(4), 318-327. http://dx.doi.org/10.3109/09540261.2011.606803

Cohen, J. (1988). Statistical power analysis for the behavioral sciences (2nd ed.). Hillsdale, NJ: Erlbaum.

Cuijpers, P., van Straten, A., \& Andersson, G. (2008). Internetadministered cognitive behavior therapy for health problems: A systematic review. Journal of Behavioral Medicine, 31(2), 169-177. http://dx.doi.org/10.1007/s10865-007-9144-1

Cukrowicz, K. C., \& Joiner, T. E. (2007). Computer-based intervention for anxious and depressive symptoms in a non-clinical population. Cognitive Therapy and Research, 31(5), 677-693. http://dx.doi.org/10.1007/s10608-006-9094-x

Davies, E. B., Morriss, R., \& Glazebrook, C. (2014). Computerdelivered and web-based interventions to improve depression, anxiety, and psychological well-being of university students: A systematic review and meta-analysis. Journal of Medical Internet Research, 16(5), e130. http://dx.doi.org/10.2196/jmir. 3142

Devilly, G., \& Borkovec, T. (2000). Psychometric properties of the credibility/expectancy questionnaire. Journal of
Behavior Therapy and Experimental Psychiatry, 31(2), 73-86. http://dx.doi.org/10.1016/S0005-7916(00)00012-4

Dimidjian, S., Arch, J. J., Schneider, R. L., Desormeau, P., Felder, J. N., \& Segal, Z. V. (2016). Considering metaanalysis, meaning, and metaphor: A systematic review and critical examination of "third wave" cognitive and behavioral therapies. Behavior Therapy, 47(6), 886-905. http://dx.doi. org/10.1016/j.beth.2016.07.002

Dowd, H., Hogan, M., McGuire, B., Davis, M., Sarma, K., Fish, R., \& Zautra, A. (2015). Comparison of an online mindfulness-based cognitive therapy intervention with online pain management psychoeducation: A randomized controlled study. Clinical Journal of Pain, 31(6), 517-527. http://dx.doi.org/10.1097/AJP.0000000000000201

Duval, S., \& Tweedie, R. (2000). Trim and fill: A simple funnelplot-based method of testing and adjusting for publication bias in meta-analysis. Biometrics, 56(2), 455-463. http://dx. doi.org/10.1111/j.0006-341X.2000.00455.x

Eysenbach, G. (2005). The law of attrition. Journal of Medical Internet Research, 7(1), e11. http://dx.doi.org/10.2196/jmir. 7.1.e11

Field, A. P., \& Gillett, R. (2010). How to do a meta-analysis. British Journal of Mathematical and Statistical Psychology, 63(3), 665-694. http://dx.doi.org/10.1348/000711010X502733

Firth, N., Barkham, M., \& Kellett, S. (2015). The clinical effectiveness of stepped care systems for depression in working age adults: A systematic review. Journal of Affective Disorders, 170, 119-130. http://dx.doi.org/10.1016/j.jad.2014.08.030

Forsyth, J. P., \& Sheppard, S. C. (2009). Behavior therapy and behavior analysis: Overview and third-generation perspectives. In D. C. Richard \& S.K. Huprich (Eds.), Clinical psychology: Assessment, treatment, and research (pp. 249-280). Burlington, MA: Elsevier Academic Press.

Frisch, M. B., Cornell, J., Villanueva, M., \& Retzlaff, P. J. (1992). Clinical validation of the Quality of Life Inventory: A measure of life satisfaction of use in treatment planning and outcome assessment. Psychological Assessment, 4, 92-101. http://dx.doi.org/10.1037/1040-3590.4.1.92

Gilbert, P. (2010). Compassion focused therapy: Distinctive features. New York, NY: Routledge.

Glick, D. M., \& Orsillo, S. M. (2015). An investigation of the efficacy of acceptance based behavioral therapy for academic procrastination. Journal of Experimental Psychology: General, 144(2), 400-409. http://dx.doi.org/10.1037/ xge0000050

Graber, J. A., \& Sontag, L. M. (2009). Internalizing problems during adolescence. In R. M. Lerner \& L. Steinberg (Eds.), Handbook of adolescent psychology (3rd ed., pp. 642-682). Hoboken, NJ: Wiley.

Grist, R., \& Cavanagh, K. (2013). Computerised cognitive behavioural therapy for common mental health disorders, what works, for whom under what circumstances? A systematic review and meta-analysis. Journal of Contemporary Psychotherapy, 43(4), 243-251. http://dx.doi.org/10.1007/ s10879-013-9243-y

Hacker, T., Stone, P., \& MacBeth, A. (2016). Acceptance and commitment therapy-do we know enough? Cumulative and sequential meta-analyses of randomized controlled trials. Journal of Affective Disorders, 190, 551-565. http://dx.doi. org/10.1016/j.jad.2015.10.053

Hayes, S. C., Masuda, A., Bissett, R., Luoma, J., \& Guerrero, L. F. (2004). DBT, FAP, and ACT: How empirically oriented are the new behavior therapy technologies? Behavior Therapy, 35(1), 35-54. http://dx.doi.org/10.1016/S0005-7894(04)80003-0

Hayes, S. C., Strosahl, K. D., \& Wilson, K. G. (1999). Acceptance and commitment therapy: An experiential approach to behaviour change. London, UK: Guilford Press. 
Hayes, S. C., Villatte, M., Levin, M., \& Hildebrandt, M. (2011). Open, aware, and active: Contextual approaches as an emerging trend in the behavioral and cognitive therapies. Annual Review of Clinical Psychology, 7, 141-168. http://dx.doi.org/10.1146/annurev-clinpsy-032210-104449

Hesser, H., Gustafsson, T., Lundén, C., Henrikson, O., Fattahi, K., Johnsson, E., . . . Andersson, G. (2012). A randomized controlled trial of internet-delivered cognitive behavior therapy and acceptance and commitment therapy in the treatment of tinnitus. Journal of Consulting and Clinical Psychology, 80(4), 649. http://dx.doi.org/10.1037/a0027021

Higgins, J. P., Altman, D. G., \& Sterne, J. A. (2011). Assessing risk of bias in included studies. In J. P. Higgins \& S. Green (Eds.), Cochrane handbook for systematic reviews of interventions: Version 5.1.0. Retrieved from www.cochrane-handbook.org.

Hofmann, S. G., Sawyer, A. T., \& Fang, A. (2010). The empirical status of the "new wave" of cognitive behavioral therapy. Psychiatric Clinics of North America, 33(3), 701-710. http://dx.doi.org/10.1016/j.psc.2010.04.006

Hudson, C. G. (2005). Socioeconomic status and mental illness: Tests of the social causation and selection hypotheses. American Journal of Orthopsychiatry, 75(1), 3-18. http://dx.doi.org/10. 1037/0002-9432.75.1.3

Hunot, V., Moore, T. H., Caldwell, D. M., Furukawa, T. A., Davies, P., Jones, H., . . . Churchill, R. (2013). "Third wave” cognitive and behavioural therapies versus other psychological therapies for depression. Cochrane Database of Systematic Reviews, 10. http://dx.doi.org/10.1002/14651858.CD008704. pub2

Internet World Stats. (2016, June 30). World internet usage and population statistics. Retrieved from http://www.internetworldstats.com/stats.htm.

Jacob, G. A., \& Arntz, A. (2013). Schema therapy for personality disorders: A review. International Journal of Cognitive Therapy, 6(2), 171-185. http://dx.doi.org/10.1521/ijct.2013. 6.2.171

Jacobson, N. S., \& Christensen, A. (1996). Integrative couple therapy: Promoting acceptance and change. New York, NY: Norton.

Kabat-Zinn, J. (1990). Full catastrophe living: Using the wisdom of your body and mind to face stress, pain and illness. New York, NY: Random House.

Kahl, K. G., Winter, L., \& Schweiger, U. (2012). The third wave of cognitive behavioural therapies: What is new and what is effective? Current Opinion in Psychiatry, 25(6), 522-528. http://dx.doi.org/10.1097/YCO.0b013e328358e531

Kelly, A., \& Carter, J. (2015). Self-compassion training for binge eating disorder: A pilot randomized controlled trial. Psychology and Psychotherapy: Theory, Research and Practice, 88(3), 285-303. http://dx.doi.org/10.1111/papt.12044

Kenardy, J., McCafferty, K., \& Rosa, V. (2003). Internetdelivered indicated prevention for anxiety disorders: A randomized controlled trial. Behavioural and Cognitive Psychotherapy, 31, 279-289. http://dx.doi.org/10.1017/ S1352465803003047

Kivi, M., Eriksson, M., Hange, D., Petersson, E. L., Vernmark, K., Johansson, B., \& Björkelund, C. (2014). Internet-based therapy for mild to moderate depression in Swedish primary care: Short term results from the PRIM-NET randomized controlled trial. Cognitive Behaviour Therapy, 43(4), 289-298. http://dx.doi.org/10.1080/16506073.2014. 921834

Kliem, S., Kröger, C., \& Kosfelder, J. (2010). Dialectical behavior therapy for borderline personality disorder: A meta-analysis using mixed-effects modeling. Journal of Consulting and Clinical Psychology, 78(6), 936-951. http://dx.doi.org/10. 1037/a0021015
Kohlenberg, R. J., \& Tsai, M. (1991). Functional analytic psychotherapy: Creating intense and curative therapeutic relationships. New York, NY: Plenum.

Kristjánsdóttir, O. B., Fors, E. A., Eide, E., Finset, A., Stensrud, T. L., Van Dulmen, S., . . Eide, H. (2013). A smartphonebased intervention with diaries and therapist-feedback to reduce catastrophizing and increase functioning in women with chronic widespread pain: Randomized controlled trial. Journal of Medical Internet Research, 15(1), e5. http://dx. doi.org/10.2196/jmir.2249

Lappalainen, P., Granlund, A., Siltanen, S., Ahonen, S., Vitikainen, M., Tolvanen, A., \& Lappalainen, R. (2014). ACT internet-based vs face-to-face? A randomized controlled trial of two ways to deliver acceptance and commitment therapy for depressive symptoms: An 18-month follow-up. Behaviour Research and Therapy, 61, 43-54. http://dx.doi. org/10.1016/j.brat.2014.07.006

Lappalainen, P., Langrial, S., Oinas-Kukkonen, H., Tolvanen, A., \& Lappalainen, R. (2015). Web-based acceptance and commitment therapy for depressive symptoms with minimal support: A randomized controlled trial. Behavior Modification, 39(6), 805-834. http://dx.doi.org/10.1177/ 0145445515598142

Leaviss, J., \& Uttley, L. (2015). Psychotherapeutic benefits of compassion-focused therapy: An early systematic review. Psychological Medicine, 45, 927-945. http://dx.doi.org/10. 1017/S0033291714002141

Lee, E. B., An, W., Levin, M. E., \& Twohig, M. P. (2015). An initial meta-analysis of Acceptance and Commitment Therapy for treating substance use disorders. Drug and Alcohol Dependence, 155, 1-7. http://dx.doi.org/10.1016/j. drugalcdep.2015.08.004

Levin, M., Pistorello, J., Seeley, J., \& Hayes, S. (2014). Feasibility of a prototype web-based acceptance and commitment therapy prevention program for college students. Journal of American College Health, 62(1), 20-30. http://dx.doi.org/10. 1080/07448481.2013.843533

Linehan, M. M. (1993). Cognitive-behavioural treatment of borderline personality disorder. New York, NY: Guilford Press.

Ljótsson, B., Andersson, G., Andersson, E., Hedman, E., Lindfors, P., Andréewitch, S., . . . Lindefors, N. (2011). Acceptability, effectiveness, and cost-effectiveness of internet-based exposure treatment for irritable bowel syndrome in a clinical sample: A randomized controlled trial. BMC Gastroenterology, 11(1), 110. http://dx.doi.org/10.1186/1471-230X-11-110

Ljótsson, B., Falk, L., Vesterlund, A., Hedman, E., Lindfors, P., Rück, C., . . . Andersson, G. (2010). Internet-delivered exposure and mindfulness based therapy for irritable bowel syndrome: A randomized controlled trial. Behaviour Research and Therapy, 48(6), 531-539. http://dx.doi.org/ 10.1016/i.brat.2010.03.003

Ljótsson, B., Hedman, E., Andersson, E., Hesser, H., Lindfors, P., Hursti, T., . . . Andersson, G. (2011). Internet-delivered exposure-based treatment vs. stress management for irritable bowel syndrome: A randomized trial. American Journal of Gastroenterology, 106(8), 1481-1491. http://dx.doi.org/10. 1038/ajg.2011.139

Lovibond, S. H., \& Lovibond, P. F. (1995). Manual for the Depression Anxiety Stress Scales (2nd ed.). Sydney, Australia: Psychology Foundation of Australia.

Ly, K. H., Trüschel, A., Jarl, L., Magnusson, S., Windahl, T., Johansson, R., . . . Andersson, G. (2014). Behavioural activation versus mindfulness-based guided self-help treatment administered through a smartphone application: A randomised controlled trial. BMJ Open, 4, e003440. http://dx.doi.org/10. 1136/bmjopen-2013-003440 
McCullough, J. P. (2000). Treatment for chronic depression: Cognitive behavioural analysis system of psychotherapy (CBASP). New York, NY: Guilford Press.

Mendonça, S., Crespo, N., \& Simões, N. (2015). Inequality in the network society: An integrated approach to ICT access, basic skills, and complex capabilities. Telecommunications Policy, 39, 192-207. http://dx.doi.org/10.1016/j.telpol.2014. 12.010

Mitchell, N., \& Gordon, P. K. (2007). Attitudes towards computerized CBT for depression amongst a student population. Behavioural and Cognitive Psychotherapy, 35, 421-430. http://dx.doi.org/10.1017/S1352465807003700

Moher, D., Liberati, A., Tetzlaff, J., Altman, D. G., \& PRISMA Group. (2009). Preferred reporting items for systematic reviews and meta-analyses: The PRISMA statement. PLOS Medicine, 6(7), e1000097. http://dx.doi.org/10.1371/journal. pmed.1000097

Negt, P., Brakemeier, E. L., Michalak, J., Winter, L., Bleich, S., \& Kahl, K. G. (2016). The treatment of chronic depression with cognitive behavioral analysis system of psychotherapy: A systematic review and meta-analysis of randomizedcontrolled clinical trials. Brain and Behavior, 6(8), e00486. http://dx.doi.org/10.1002/brb3.486

Normann, N., Van Emmerik, A. A., \& Morina, N. (2014). The efficacy of metacognitive therapy for anxiety and depression: A meta-analytic review. Depression and Anxiety, 31(5), 402-411. http://dx.doi.org/10.1002/da.22273

Oh, H., Rizo, C., Enkin, M., \& Jadad, A. (2005). What is eHealth (3): A systematic review of published definitions. Journal of Medical Internet Research, 7(1), e1. http://dx.doi. org/10.2196/jmir.7.1.e1

Öst, L. G. (2008). Efficacy of the third wave of behavioral therapies: A systematic review and meta-analysis. Behaviour Research and Therapy, 46(3), 296-321. http://dx.doi.org/ 10.1016/j.brat.2007.12.005

Panos, P. T., Jackson, J. W., Hasan, O., \& Panos, A. (2014). Meta-analysis and systematic review assessing the efficacy of dialectical behavior therapy (DBT). Research on Social Work Practice, 24, 213-223. http://dx.doi.org/10.1177/ 1049731513503047

Patrick, D. L., Drossman, D. A., Frederick, I. O., DiCesare, J., \& Puder, K. L. (1998). Quality of life in persons with irritable bowel syndrome (development and validation of a new measure). Digestive Diseases and Sciences, 43(2), 400-411. http://dx.doi.org/10.1023/A:1018831127942

Pew Research Center. (2012, April 13). Digital differences. Retrieved from http://www.pewinternet.org/2012/04/13/ digital-differences/.

Pierce, B., Twohig, M., \& Levin, M. (2016). Perspectives on the use of acceptance and commitment therapy related mobile apps: Results from a survey of students and professionals. Journal of Contextual Behavioral Science, 5(4), 215-224. http://dx.doi.org/10.1016/j.jcbs.2016.08.001

Piet, J., \& Hougaard, E. (2011). The effect of mindfulness-based cognitive therapy for prevention of relapse in recurrent major depressive disorder: A systematic review and meta-analysis. Clinical Psychology Review, 31, 1032-1040. http://dx.doi. org/10.1016/j.cpr.2011.05.002

Pots, W., Fledderus, M., Meulenbeek, P., Klooster, P., Schreurs, K., \& Bohlmeijer, E. (2015). Acceptance and commitment therapy as a web-based intervention for depressive symptoms: Randomised controlled trial. British Journal of Psychiatry, 208(1), 69-77. http://dx.doi.org/10. 1192/bjp.bp.114.146068

Proudfoot, J., Klein, B., Barak, A., Carlbring, P., Cuijpers, P., Lange, A., ... Andersson, G. (2011). Establishing guidelines for executing and reporting internet intervention research.
Cognitive Behaviour Therapy, 40(2), 82-97. http://dx.doi. org/10.1080/16506073.2011.573807

Radloff, L. S. (1977). The CES-D scale: A self-report depression scale for research in the general population. Applied Psychological Measurement, 1(3), 385-401. http://dx.doi.org/10. 1177/014662167700100306

Rosen, G. M., Glasgow, R. E., Moore, T. E., \& Barrera, M. (2015). Self-help therapy: Recent developments in the science and business of giving psychology away. In S. O. Lilienfeld, S. J. Lynn, \& J. M. Lohr (Eds.), Science and pseudoscience in clinical psychology (2nd ed., pp. 245-274). New York, NY: Guilford Press.

Segal, Z. V., Williams, J. M., \& Teasdale, J. D. (2002). Mindfulness-based cognitive therapy for depression: A new approach to preventing relapse. New York, NY: Guilford Press.

Shapira, L., \& Mongrain, M. (2010). The benefits of selfcompassion and optimism exercises for individuals vulnerable to depression. Journal of Positive Psychology, 5(5), 377-389. http://dx.doi.org/10.1080/17439760.2010.516763

Spielberger, C. D., Gorsuch, R. L., Lushene, R., Vagg, P. R., \& Jacobs, G. A. (1983). Manual for the State-Trait Anxiety Inventory. Palo Alto, CA: Consulting Psychologists Press.

Spijkerman, M. P., Pots, W. T., \& Bohlmeijer, E. T. (2016). Effectiveness of online mindfulness-based interventions in improving mental health: A review and meta-analysis of randomised controlled trials. Clinical Psychology Review, 45, 102-114. http://dx.doi.org/10.1016/j.cpr.2016.03.009

Stephens, J., \& Allen, J. (2013). Mobile phone interventions to increase physical activity and reduce weight: A systematic review. Journal of Cardiovascular Nursing, 28(4), 320-329. http://dx.doi.org/10.1097/JCN.0b013e318250a3e7

Sterne, J. A., Egger, M., \& Moher, D. (2011). Addressing reporting biases. In J. P. Higgins \& S. Green (Eds.), Cochrane handbook for systematic reviews of interventions: Version 5.1.0. Retrieved from www.cochrane-handbook.org.

Svanborg, P., \& Asberg, M. (1994). A new self-rating scale for depression and anxiety states based on the comprehensive psychopathological rating scale. Acta Psychiatrica Scandinavica, 89(1), 21-28. http://dx.doi.org/10.1111/j.1600-0447. 1994.tb01480.x

Tait, R. J., Spijkerman, R., \& Riper, H. (2013). Internet and computer based interventions for cannabis use: A meta-analysis. Drug and Alcohol Dependence, 133, 295-304. http://dx.doi. org/10.1016/j.drugalcdep.2013.05.012

Trompetter, H., Bohlmeijer, E., Veehof, M., \& Schreurs, K. (2015). Internet-based guided self-help intervention for chronic pain based on acceptance and commitment therapy: A randomized controlled trial. Journal of Behavioral Medicine, 38(1), 66-80. http://dx.doi.org/10.1007/ s10865-014-9579-0

Tullis, T., \& Albert, W. (2008). Measuring the user experience. San Francisco, CA: Morgan Kaufmann.

Valentine, J. C., Pigott, T. D., \& Rothstein, H. R. (2010). How many studies do you need? A primer on statistical power for meta-analysis. Journal of Educational and Behavioral Statistics, 35(2), 215-247. http://dx.doi.org/10.3102/ 1076998609346961

Ware, J., Kosinski, M., Dewey, J., \& Gandek, B. (2001). How to score and interpret single-item health status measures: Manual for users of the SF-8 Health Survey. Boston, MA: QualityMetric.

Wells, A. (2009). Metacognitive therapy for anxiety and depression. London, UK: Guilford Press.

Weineland, S., Arvidsson, D., Kakoulidis, T., \& Dahl, J. (2012). Acceptance and commitment therapy for bariatric surgery patients: A pilot RCT. Obesity Research and Clinical Practice, 6(1), e21-e30. http://dx.doi.org/10.1016/j.orcp.2011.04.004 
WHOQOL Group. (1998). Development of the World Health Organization WHOQOL-BREF Quality of Life Assessment. Psychological Medicine, 28(3), 551-558. http://dx.doi.org/ $10.1017 /$ S0033291798006667

Young, J. E. (1990). Cognitive therapy for personality disorders: A schema focused approach. Sarasota, FL: Professional Resource Exchange.
Zigmond, A. S., \& Snaith, R. P. (1983). The Hospital Anxiety and Depression Scale. Acta Psychiatrica Scandinavica, 67, 361-370. http://dx.doi.org/10.1111/j.1600-0447.1983.tb09716.x

Received: March 17, 2017

ACCEPTED: July 21, 2017

Available online: $\mathbf{x x x}$ 